\title{
Inserción de implantes dentales en la apófisis pterigoides: Una alternativa en el tratamiento rehabilitador del maxilar posterior atrófico
}

\author{
MATEOS L * \\ GARCIA-CALDERON M ** \\ GONZALEZ-MARTIN M * \\ GALLEGO D * \\ CABEZAS J *
}

\begin{abstract}
Mateos L, García-Calderón M, González-Martín M, Gallego D, Cabezas J. Inserción de implantes dentales en la apófisis pterigoides: Una alternativa en el tratamiento rehabilitador del maxilar posterior atrófico. Av Periodon Implantol. 2002; $14,1: 37-45$.
\end{abstract}

\begin{abstract}
RESUMEN
Existe una alta tasa de fracasos en el tratamiento con implantes del maxilar superior reabsorbido, así como aquéllos insertados en zonas de hueso tipo IV (hueso cortical delgado que rodea a hueso trabecular de baja densidad). Sin embargo, existe una mayor predictibilidad en la colocación de implantes en la apófisis pterigoides, justificando sus indicaciones en aquellos casos donde existe un pronóstico incierto si se insertan las fijaciones en un maxilar de las características antes mencionadas. El uso de implantes en la apófisis pterigoides tienen una tasa de éxito similar a la de los implantes usados en otras áreas del maxilar superior, proporcionando una importante ayuda a la estabilización de la prótesis sobre implantes, lo que otorga una ventaja biomecánica a este tipo de rehabilitaciones.

El objetivo de este trabajo es realizar una revisión sobre el procedimiento y la anatomía de la zona, analizando los conceptos de anatomía aplicada del maxilar posterior a la terapéutica con implantes y desarrollando un protocolo quirúrgico para la inserción de fijaciones intraóseas en la apófisis pterigoides, estableciendo las consideraciones protésicas para la carga de los implantes situados en esta región.

El protocolo quirúrgico incluye el anclaje cortical del implante en la lámina pterigoidea. Para alcanzar este objetivo, el implante deberá insertarse con una cierta angulación respecto al plano oclusal, relacionándose en su colocación con los huesos maxilar superior, palatino, y la apófisis pterigoides del hueso esfenoides. Por su parte, el protocolo protésico también presenta variaciones, como la toma de impresiones del implante en vez del pilar transepitelial, entre otros. Estos cambios favorecen las condiciones biomecánicas y funcionales de los implantes, haciendo posible el éxito a largo plazo de los mismos.
\end{abstract}

\section{PALABRAS CLAVE}

Implantes dentales, apófisis pterigoides, maxilar atrófico.

\section{INTRODUCCIÓN}

La literatura ha documentado ampliamente el que los implantes dentales oseointegrados poseen una alta predictibilidad a medio y a largo plazo, tanto en el tratamiento de pacientes totalmente desdentados como en los parcialmente desdentados. Sin embargo, en todos los estudios encontramos un porcentaje variable de fracasos, debido a diferentes problemas (1-4).

\footnotetext{
* Odontólogo.

** Médico Estomatólogo. Doctor en Odontología.
} 
Diversos autores han publicado estudios sobre la efectividad de los implantes, intentando relacionar las características de los pacientes, el estado periodontal, y otros factores como la longitud y diámetro de las fijaciones, con el número de fracasos obtenidos en cada uno de los estudios realizados. Ya VAN STEENBERGHE, M.D., LEKHOLM, U. y cols. (5) relacionan un mayor número de fallos con las siguientes circunstancias: una calidad ósea menos favorable (tipo III y IV tal como los definen LEKHOLM, U. y ZARB, G.A. (6), un mayor índice de placa bacteriana antes de la colocación del implante, y con unas fijaciones de menor diámetro y longitud. Estas causas de fracaso son corroboradas en el mismo estudio a los 3 años de seguimiento (7). En la misma línea de trabajo, en 1991, JAFFIN, R.A. y BERMAN, C.L. (8) señalan que existe un mayor número de fracasos en zonas de hueso de calidad tipo IV de la clasificación citada previamente, que corresponde a hueso cortical delgado que rodea a hueso trabecular de baja densidad, el cual se encuentra muy frecuentemente en el maxilar posterior.

También son recogidas en la literatura otras características del maxilar superior en sus sectores posteriores, que hacen que se observe un mayor número de fracasos en los implantes colocados en esta región, tales como su morfología, problemas de accesibilidad y dificultad en la higiene bucal $(7,8)$.

A estas peculiaridades anatómicas y de acceso debemos añadir que existe una mayor carga oclusal en las regiones molares, en comparación con otras zonas del maxilar, y que este factor ocasiona que la tasa de éxito de los implantes en el maxilar posterior sea menor que en otras zonas maxilares y mandibulares (9).

Para solucionar estos problemas, una gran variedad de procedimientos han sido descritos en la literatura, con la intención de modificar y mejorar estos factores anatómicos y biomecánicos. Entre ellos destaca la elevación del suelo del seno maxilar ("sinus lift") como técnica de referencia propuesta en numerosos trabajos (10-14). No obstante, esta opción presenta también algunas limitaciones y cierta complejidad quirúrgica; en 1996, GARCÍA-CALDERÓN, M. y cols analizaron las ventajas y desventajas del sinus lift como técnica quirúrgica aplicada en el maxilar posterior atrófico, y analizaron las alternativas terapéuticas a este procedimiento para la rehabilitación con implantes de esta región. Entre ellas describen la inserción de implantes en regiones no habituales y en arbotantes anatómicos, combinados a veces con técnicas de regeneración ósea, así como con técnicas que proponen el aumento en el número de implantes y el uso de fijaciones de mayor diámetro en el hueso maxilar (9-15-21-24).

La inserción de fijaciones intraóseas en arbotantes anatómicos ya fue descrita por KROGH, P. en 1991 (16), donde señala la zona ósea inferior al seno maxilar, la región tuberositaria, y las apófisis piriforme y pterigoides como posibles receptoras de implantes en el maxilar superior. Por otro lado, la regeneración ósea guiada es hoy en día una técnica predecible de amplio uso en implantología, tanto en casos de edentulismo maxilar posterior como en otras muchas situaciones clínicas que buscan la inserción ideal de los implantes desde el punto de vista prostodóncico (17-20).

Ya TUSLANE, J.F., en 1989 (25), describió por primera vez la técnica de inserción de implantes en las tuberosidades maxilares, para evitar la necesidad de procedimientos de elevación de seno junto con injertos de hueso, en maxilares con escaso espacio infrasinusal debido a una hiperneumatización del seno. Este autor señala que el hueso tuberositario es frecuentemente de pobre calidad, con escaso volumen y baja densidad. Assí, defiende que los implantes situados en esta región debían llegar a la densa cortical ósea de la lámina pterigoidea, favoreciendo el anclaje primario de la fijación intraósea.

En 1991, KROGH, P.H. (16) realizó un análisis de las peculiaridades anatómicas y quirúrgicas del uso de implantes en pacientes con atrofia severa del maxilar, recomendando la colocación de éstos en los arbotantes anatómicos como el área piriforme, es decir, la zona posterior al canino y anterior a la pared anterior del seno, y la tuberosidad o lámina pterigoidea, dándole en este último caso una inclinación posterior a las fijaciones intraóseas.

Más tarde, en 1992, BALSHI, T.J. (26) documenta el uso de implantes unitarios en esta región anatómica, como soporte de prótesis implantodentosoportadas; señala las ventajas de utilizar esta técnica en los casos en los que, debido a la presencia de dientes remanentes con buen soporte periodontal anteriores al seno maxilar agrandado, el autor justifica la unión del implante a estos dientes. Se describen los procedimientos quirúrgicos y protésicos de esta técnica, y analiza las consideraciones conceptuales de la unión diente-implante, con las consiguientes peculiaridades funcionales y biomecánicas que esto conlleva, y que también son analizadas en su estudio.

También en 1992, BAHAT, O. (27) realizó un estudio sobre 45 pacientes tratados consecutivamente mediante la inserción de 72 implantes en la región tuberositaria correspondiente al tercer molar, con una tasa de éxito del $93 \%$ y un seguimiento a los 2 años, analizando la calidad y cantidad ósea del maxilar posterior, características ergonómicas, pronóstico y requisitos para una alta predictibilidad de los implantes colocados en esta región. Los factores considerados por el autor para conseguir este alto porcentaje de éxito incluyen una meticulosa identificación y prevención de 
todas las posibles complicaciones, modificación de la secuencia de fresado para maximizar la estabilización inicial del implante, conseguir, cuando sea necesario, la bicorticalización en el anclaje de las fijaciones, además de un tratamiento cuidadoso de los tejidos blandos y del periostio, y eliminación o minimización de las cargas oclusales transmucosas durante el período de oseointegración y las cargas no axiales tras la conexión de los componentes protésicos correspondientes.

GRAVES, S.L. (28), en 1994, analiza las ventajas y desventajas del uso de implantes en la apófisis pterigoides, realizando una descripción anatómica de la zona tuberositaria y pterigoidea, así como de la técnica de inserción de implantes en esta región. Acompaña a su publicación un estudio preliminar de la colocación, en 49 pacientes, de 64 fijaciones intraóseas en la lámina pterigoidea, obteniendo 7 fracasos, todos ellos en la segunda cirugía, cuando se comprobó su movilidad. El autor destaca un seguimiento de hasta 4 años en alguno de los implantes, con la característica común de no fracasar ninguno después de ser cargados con las restauraciones protésicas, lo que atribuye a una respuesta positiva de estos implantes a las fuerzas no axiales ejercidas durante la masticación, y que los diferencian, en este aspecto, de la dentición natural y de los implantes insertados en otras regiones de la cavidad oral. Este autor considera varias ventajas a esta técnica, entre ellas la preservación de la integridad del seno maxilar, sin necesidad de realizar un injerto de hueso, y evitando el uso de prótesis con extensiones distales, con lo que se consigue una mejor distribución de las fuerzas oclusales. Además, se acorta el tiempo de tratamiento global en comparación con otras técnicas de implantes, como la elevación de seno. Entre las desventajas señala la dificultad del procedimiento, al tener que introducir el implante a través de $15-20 \mathrm{~mm}$ de hueso, así como la necesidad de un adecuado soporte óseo tanto en la tuberosidad del maxilar como en la región pterigoidea.

De nuevo BALSHI, T.J. (29), en 1995, en un estudio preliminar, comunica los resultados de la colocación de 187 implantes en la región posterior del maxilar superior de 44 pacientes parcialmente edéntulos. De estos 187 implantes, 51 fueron insertados en el área pterigomaxilar, con una tasa de éxito del $86.3 \%$ tras un período medio de carga de 12.6 meses. En este estudio se analizan los factores biológicos y biomecánicos relacionados con estos 51 implantes, tales como el procedimiento quirúrgico, el período de oseointegración, el manejo de los tejidos blandos y el tipo de hueso receptor de los implantes (según su cantidad y calidad), así como la confección de los componentes protésicos necesarios para la carga funcional de los mismos. En este trabajo sólo se investigaron los implantes colocados en la apófisis pterigoides de pacientes parcialmente edéntulos, por lo que se con- sideraron las complicaciones biomecánicas y el riesgo potencial que supone unir dientes naturales a implantes oseointegrados.

En 1998, PI-URGELL, J. presentó un estudio retrospectivo sobre 177 implantes insertados en la región pterigomaxilar de 136 pacientes (30); de estos implantes, 95 tuvieron un seguimiento entre 5 a 10 años, mientras que el resto fueron monitorizados durante 1 a 5 años. Las restauraciones protésicas realizadas sobre estos implantes incluyeron 144 prótesis fijas parciales y 17 totales. El porcentaje de éxito que obtuvo el autor fue del $97.2 \%$, tras el fracaso de 4 fijaciones no osteointegradas y retiradas en la segunda cirugía, además de un implante que se perdió tras la oseointegración por fractura tras tres años de carga.

En este artículo realizaremos una revisión de la literatura referente a la inserción de implantes en la apófisis pterigoides, además de un análisis anatómico descriptivo de dicha área, describiendo un protocolo de inserción de implantes en el área pterigoidea.

\section{ANATOMÍA DEL ÁREA}

Los distintos estudios anatómicos sobre esta región nos ofrecen la clave para el entendimiento de la complejidad de estructuras que se dan cita en esta zona del organismo (31-38); estos trabajos indican que el implante que se ancle en la cortical pterigoidea se relaciona, en su inserción con tres huesos: la apófisis pterigoides del hueso esfenoides, el hueso maxilar y el hueso palatino (Figs. 1 y 2 ).

Las apófisis pterigoides son dos pequeñas columnas óseas, situadas en la parte inferior del hueso esfenoides. Están verticalmente dirigidas de arriba a abajo; debemos considerar en ellas dos raíces, que se pro-

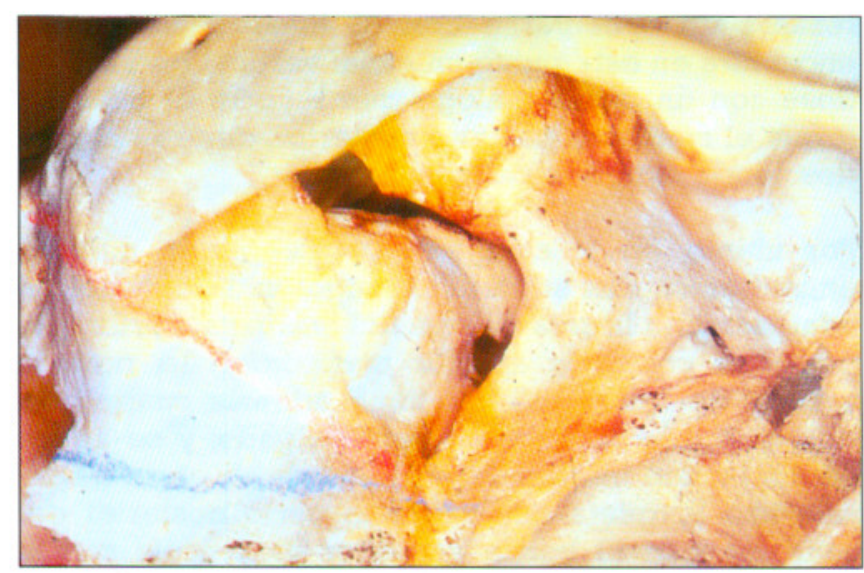

Fig. 1: Área de inserción del implante pterigoideo. Se relaciona en la colocación con el maxilar superior, el hueso palatino, y la apófisis pterigoides del hueso esfenoides. 


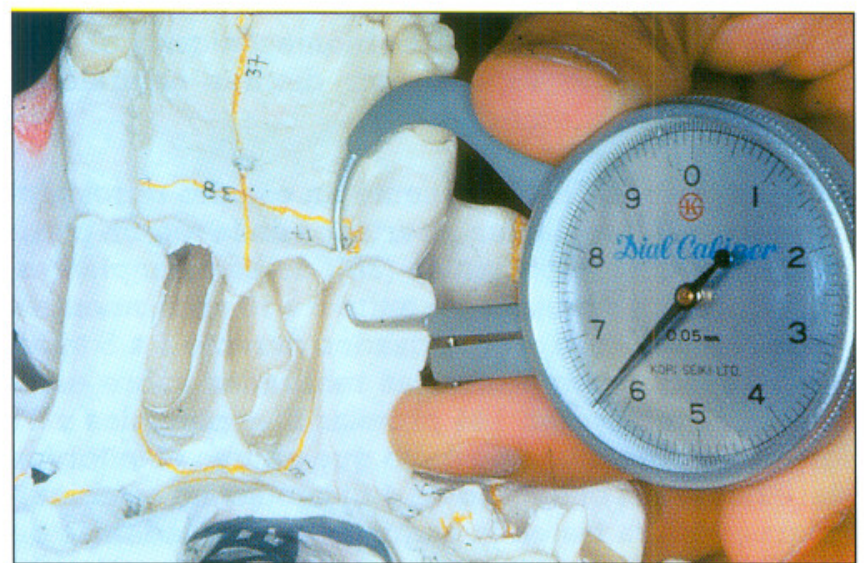

Fig. 2: Zona densa de hueso cortical (de unos $6 \mathrm{~mm}$ de espesor) que dará soporte al implante. Se encuentra en la región medial de la apófisis pterigoides entre las láminas, localizada a 3 o $4 \mathrm{~mm}$ de la cresta alveolar en dirección medial.

longan hacia abajo por hojas aisladas por detrás y unidas por delante; son las alas interna y externa de la apófisis pterigoides, que comprenden en dicho espacio la fosa pterigoidea. Los bordes anteriores de las dos alas se reúnen y forman en la apófisis pterigoides la cara anterior, que forma en la parte más superior el límite posterior de la entrada del fondo de la fosa pterigomaxilar. En su parte inferior, la cara anterior se escota, es decir, las dos alas recobran aquí su individualidad; de ello resulta una escotadura en la que viene a articularse la apófisis piramidal del palatino. Superiormente a esta unión se encuentra la fosa pterigopalatina, que contiene la porción terminal de la arteria maxilar.

El maxilar superior posee cuatro bordes; el borde posterior, el de mayor interés en nuestro estudio, es redondeado y muy grueso, y recibe el nombre de tuberosidad del maxilar; es liso en su mitad superior, donde constituye la pared anterior de la fosa pterigomaxilar, y en su mitad inferior está cubierto de asperezas para articularse con el hueso palatino. Se encuentra en este punto un canal vertical que, uniéndose con un canal análogo que recorre el palatino, forma el conducto palatino posterior, dentro del cual desciende el nervio palatino anterior.

Por último, en la cara externa del hueso palatino encontramos dos superficies rugosas: la anterior se adapta contra la cara interna del maxilar superior, y la posterior contra la apófisis pterigoides. La porción vertical del hueso palatino tiene, además, cuatro bordes; el borde anterior es muy delgado, y se aplica contra la cara interna del maxilar; de su parte media sale una lengüeta que cubre la parte posterior del seno maxilar. El borde posterior se articula con la cara interna de la apófisis pterigoides. Del borde inferior se desprende una apófisis, la apófisis piramidal del palatino, que se dirige hacia atrás y hacia afuera, en dirección al espacio que queda entre las dos alas de la apófisis pterigoides.

\section{PROTOCOLO QUIRÚRGICO}

La inserción de implantes en la región pterigoidea es una técnica que requiere experiencia quirúrgica y un conocimiento anatómico preciso de la zona, debido a las estructuras nobles que rodean al área de inserción del implante y a la propia complejidad de la técnica de la cirugía de implantes. Por ello necesita de un minucioso estudio del caso y de un diagnóstico radiológico correcto. La fijación insertada pasaría a través de una zona de hueso compuesta por la maxila, hueso palatino y anclaje en la lámina pterigoidea del hueso esfenoides, con una angulación hacia distal, siguiendo un protocolo de inserción que a continuación definiremos.

En la selección de los pacientes debemos considerar, del mismo modo que en la inserción de implantes en otras regiones anatómicas, tanto la condición general de salud del paciente como las lesiones localizadas en el medio oral, que deberán ser tratadas previamente. También debemos considerar los aspectos morfológicos de la zona que vamos a intervenir; tal como hemos recogido en nuestro estudio de anatomía aplicada, el riesgo de dañar estructuras anatómicas significativas al insertar los implantes en la apófisis pterigoides es mínimo, siempre que se siga un protocolo quirúrgico correcto; una rama del nervio alveolar superior posterior pasa entre las láminas pterigoideas, ocupando los músculos pterigoideos la mayor parte del espacio entre estas láminas óseas. Por su parte, la arteria maxilar interna cruza $1 \mathrm{~cm}$ por encima de la sutura pterigomaxilar cuando entra en la fosa pterigopalatina, porción más superior y posterior de la fosa pterigomaxilar, siendo por tanto la distancia desde esta arteria al límite inferior de la sutura pterigomaxilar de $25 \mathrm{~mm}$, lo que hace de esta zona un área de trabajo segura para el cirujano de implantes, debido a la ausencia de estructuras vitales en el área de inserción de la fijación. De este modo, el posible sangrado que nos podamos encontrar en esta región se deberá seguramente a las venas del músculo pterigoideo interno, que se detiene rápidamente cuando se inserta la fijación intraósea, sin reactivarse la hemorragia una vez el implante está estabilizado.

La mala calidad ósea, factor decisivo para conseguir la correcta oseointegración de los implantes, se determinará a partir del análisis radiográfico presente en nuestro plan de tratamiento inicial, ayudado por la sensación táctil del cirujano durante el fresado en el momento de la intervención quirúrgica. Como norma general en el maxilar superior, está ya establecido el concepto de no usar implantes menores de $10 \times 3.75$ 
mm. en huesos de calidad tipo III o tipo IV, eliminando así el efecto negativo de la calidad ósea sobre la predictibilidad de los implantes. A este respecto, y en el área que nos ocupa, esta baja calidad en el hueso remanente puede compensarse mediante el anclaje cortical de la fijación intraósea en la apófisis pterigoides, lo que nos obligará a utilizar implantes de una longitud de 13-15 mm. como mínimo.

Por otra parte, el anclaje cortical del implante en la lámina pterigoidea es esencial para la estabilización inicial y el éxito a largo plazo. La zona de hueso cortical denso que dará soporte al implante se encuentra en la región medial de la apófisis pterigoides entre las láminas, localizada a 3 o $4 \mathrm{~mm}$ de la cresta alveolar en dirección medial, por lo que el implante deberá insertarse con una ligera angulación medial para alcanzar esta densa capa cortical (de unos $6 \mathrm{~mm}$ de espesor). Por lo tanto, el implante tendrá, si atraviesa este pilar de hueso con una angulación de 45 grados, de 8 a 9 $\mathrm{mm}$ de hueso cortical denso, lo cual garantizará su estabilización primaria y aumentará su predictibilidad.

Precisamente esta angulación de 45 grados es lo que evita que interfiera con el seno maxilar en su porción inferior y posterior. De hecho, es la posición del seno, junto con la necesidad del anclaje cortical y los problemas de accesibilidad, lo que determina esta angulación del implante. Aún así, si durante el fresado observamos que la membrana de la cavidad sinusal ha sido perforada, se realizará un nuevo alojamiento para el implante, que estará situado al menos $3 \mathrm{~mm}$. posterior a la localización previa.

Por último, debemos considerar los factores ergonómicos, que afectan al procedimiento quirúrgico, ya que la dificultad de acceso a la tuberosidad y a la región pterigoidea hace más complicada la inserción de implantes en esta región.

\section{Secuencia quirúrgica (figs. 3-5)}

Tras un correcto estudio radiológico, que aportará la información necesaria sobre el grado de neumatización del seno, la forma de la tuberosidad y la densidad del hueso y tamaño de la lámina pterigoidea.

Los procedimientos quirúrgicos se llevan a cabo generalmente bajo una premedicación y anestesia local, realizando una anestesia por bloqueo del nervio alveolar superior posterior, además de una anestesia por infiltración de los nervios palatino posterior, medio y anterior. También podemos realizar una anestesia por infiltración de la zona vestibular, inyectando el anestésico posterolateralmente a la tuberosidad. Los efectos anestésicos conseguidos por infiltra-

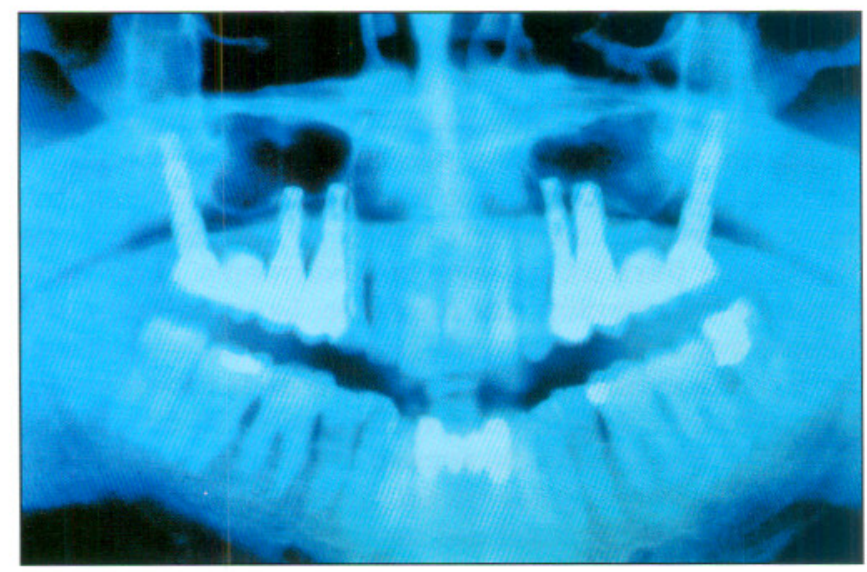

Fig. 3: Implantes dentales insertados en ambas apófisis pterigoides, lo que proporciona una excelente estabilidad biomecánica al tratamiento rehabilitador, ya que evita las extensiones distales de la prótesis.

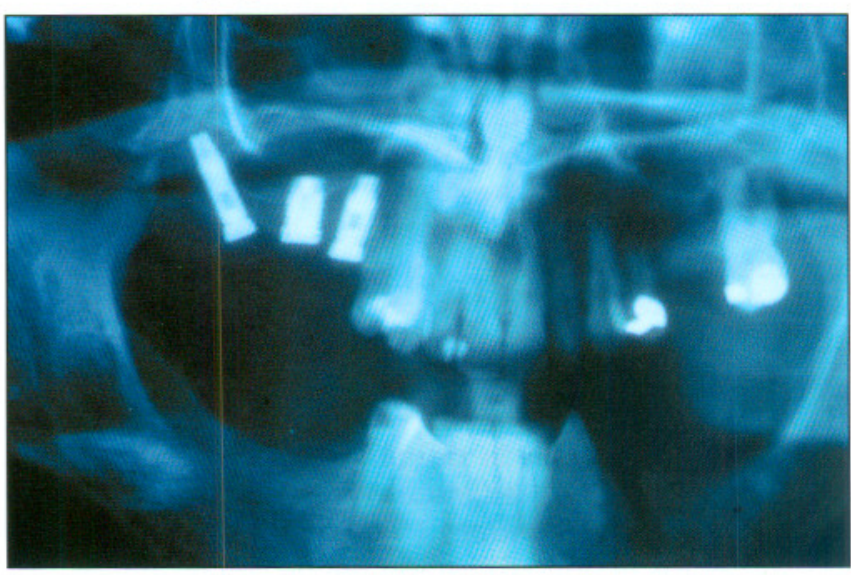

Fig. 4: Implantes insertados como alternativa al sinus lift. Se colocaron dos implantes por debajo del seno maxilar y uno en la región pterigoidea.

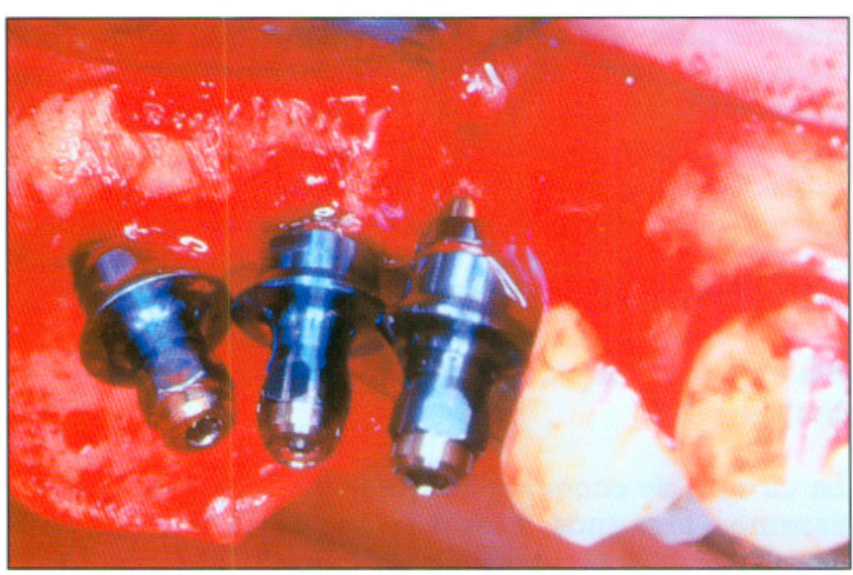

Fig. 5: En el acto quirúrgico, la inserción angulada del implante pterigoideo (en la posición más distal) es esencial para conseguir el anclaje cortical de la fijación.

ción y por bloqueo son los mismos, si bien con esta última técnica éstos son más predecibles, al no necesitar la difusión ósea de la solución anestésica. 
Se realiza una incisión supracrestal media, que debemos extender hasta la parte más posterior de la tuberosidad desde la región premolar, con una incisión de descarga vertical a este nivel anterior; despegamos un colgajo mucoperióstico hacia el fondo del vestíbulo, exponiendo completamente la tuberosidad, de modo que permita trabajar cómodamente al cirujano durante la inserción del implante. La angulación del mismo vendrá determinada por el piso del seno maxilar y por la altura vertical del hueso de la tuberosidad, de modo que, usualmente, variará entre 35-55 grados, de forma que el área de acceso al tornillo que unirá la futura prótesis al pilar transepitelial se encontrará a nivel de la cara oclusal del segundo molar.

Una vez estimada la dirección que tomará el implante, realizamos el fresado del lecho; en este procedimiento, palpamos el proceso hamular con el dedo índice de la mano que ayuda y dirigimos el fresado 3$5 \mathrm{~mm}$ lateralmente y con una angulación aproximada de 45 grados respecto al plano oclusal. El proceso hamular es la principal referencia que tenemos para determinar la localización de la lámina pterigoidea; si la vía de inserción es correcta, la fresa encontrará un hueso cortical denso a unos 10-15 mm de profundidad, donde notaremos que la fresa irá disminuyendo su velocidad, debido precisamente a la mayor densidad del hueso existente en esta región.

Por último, debido a la delgada cortical existente en esta zona (a veces inexistente) recomendamos no utilizar la fresa avellanadora ("countersink"), con lo que favorecemos la inmovilización inicial del implante, por impactación de la cabeza del implante sobre el lecho óseo. Igualmente, y debido a la pobre calidad del hueso trabecular de la zona tuberositaria, se recomienda usar fijaciones autorroscantes que eliminen la necesidad de la realización de la rosca en el lecho óseo (aterrajado). Si el implante se ha insertado correctamente, la cabeza del mismo deberá quedar impactada en la cortical del hueso tuberositario al mismo nivel de la cresta ósea, como hemos referido anteriormente. Algunos autores recomiendan que el implante exceda de 3 a $4 \mathrm{~mm}$ al traspasar la cortical pterigoidea dentro de la fosa pterigoidea (28). Finalmente, adaptamos los colgajos y suturamos la herida.

La cirugía de conexión del pilar transepitelial la realizaremos, al menos, a los 6 meses de la primera cirugía. Para ello, elevaremos un colgajo por medio de una incisión supracrestal palatinizada, que nos dará el acceso a la parte coronal del implante, y nos permitirá retirar el tornillo de cierre que protege la rosca interna de la fijación intraósea. Tras comprobar el éxito de la oseointegración con una fuerza de torque de $20 \mathrm{~N} / \mathrm{cm}$, colocamos un pilar de cicatrización de altura adecuada. En esta segunda cirugía remode- lamos los tejidos blandos para facilitar la higiene y mejorar la estética, a pesar de que es una zona donde los aspectos estéticos no son tan importantes como en la región anterior. Debemos reducir en esta fase el espesor de estos tejidos blandos hasta un máximo de 3-4 mm, suturando de nuevo el colgajo con sutura no reabsorbible y puntos simples o de colchonero. Entre 7 y 10 días después de la segunda cirugía, retiramos los puntos de sutura y comprobamos el ajuste del pilar transepitelial de cicatrización.

\section{CONSIDERACIONES EN LA FASE PROTÉSICA}

El protocolo protésico también presenta variaciones, debido a las dificultades de acceso, a la angulación del implante en su inserción y a la complejidad del procedimiento. Estos cambios favorecen las condiciones biomecánicas y funcionales de los implantes, haciendo posible el éxito a largo plazo de los mismos. Así, la inserción angulada de la fijación intraósea determina que tomemos la impresión directamente del implante, y no del pilar transepitelial; esto nos aportará datos más exactos para la posterior elección del pilar transepitelial definitivo (Fig 6).

Para la elección del pilar definitivo observamos la morfología de los tejidos blandos, el espacio interoclusal, la angulación del implante, la profundidad del surco y los factores estéticos e higiénicos, con objeto de elegir el pilar más adecuado a nuestra situación clínica. Normalmente, elegiremos un pilar estándar (cónico o cilíndrico), a menos que la angulación del implante haya sido mayor de $60^{\circ}$, en cuyo caso usaremos un pilar angulado para corregir la dirección y hacer posible la reconstrucción protésica.

En la cita de prueba de estructura de metal colada, atornillaremos ésta a los pilares y comprobaremos, visual y radiológicamente, la existencia de un perfec-

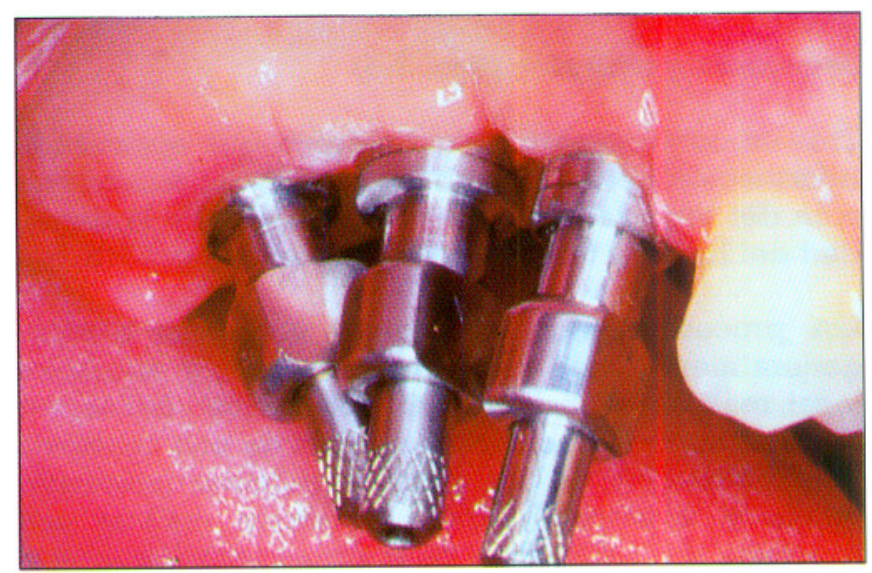

Fig. 6: Fase protésica. La toma de impresiones se realiza directamente del implante. 
to ajuste pasivo, premisa fundamental para no crear tensiones en los implantes. Además, analizaremos el diseño, que debe ser robusto a nivel de las uniones interproximales, para evitar las fracturas y las posibles flexiones de las estructuras, debido a que en esta zona las fuerzas masticatorias serán mayores que en otras regiones del maxilar. Del mismo modo, evaluaremos la presencia de un acceso adecuado con fines higiénicos, que garantice el mantenimiento en salud de los tejidos periimplantarios.

También debemos evaluar en esta fase la relación entre la superficie gingival de la prótesis y los tejidos mucosos, buscando en general un tipo de diseño "póntico-higiénico" que asegure un acceso adecuado para el mantenimiento, en un intento por compensar los problemas higiénicos causados por la accesibilidad en los sectores superiores posteriores por parte del paciente.

Finalmente, debemos instaurar un programa de mantenimiento adecuado a este tipo de tratamiento complejo, donde poder comprobar el estado de salud oral de los tejidos periimplantarios en contacto con la prótesis, determinando la presencia de mucositis periimplantaria y/o periimplantitis, la estabilidad de la prótesis y revisando la oclusión. Asimismo, realizaremos en estas citas reevaluaciones de los niveles de higiene de los pacientes, reforzando las normas de cepillado para conseguir un estado de salud oral necesario para el mantenimiento a largo plazo de las prótesis tejido-integradas.

\section{CONCLUSIONES}

- La tasa de fracasos de la terapéutica con implantes en los sectores posteriores del maxilar superior es más alta que en otras localizaciones de la cavidad oral, según los datos recogidos en la literatura. Asimismo, el tratamiento con implantes en el maxilar posterior atrófico, debido a las características especiales de éste, requiere un análisis específico de los diversos procedimientos para elegir el más adecuado según el caso.

- La inserción de implantes en la región pterigoidea puede, dada su alta predictibilidad, resolver estos problemas derivados de la atrofia maxilar sin necesidad de realizar elevaciones del suelo sinusal, evitando tomar injertos óseos y/o utilizar materiales aloplásticos de relleno, además de aportar una solución al efecto negativo de sobrecarga oclusal que se produce con el uso de prótesis con extensiones distales.

- Esta técnica es un procedimiento complejo, que requiere habilidad quirúrgica y un preciso conocimiento anatómico del área a tratar; estos conceptos, junto con una minuciosa fase diagnóstica, clínica y radiológica, son de especial relevancia en este tipo de terapéutica. De hecho, el riesgo de dañar estructuras anatómicas significativas al insertar los implantes en la apófisis pterigoides es mínimo, siempre que se siga un protocolo quirúrgico correcto.

- Si se sigue un protocolo quirúrgico adecuado, se disminuyen los riesgos y las complicaciones del tratamiento, aumentando la predictibilidad de las fijaciones intraóseas insertadas en esta región. El protocolo protésico presenta algunas variaciones, debido a la angulación del implante en su inserción y a la complejidad del procedimiento. Estas variaciones favorecen las condiciones biomecánicas y funcionales de los implantes, haciendo posible el éxito a largo plazo de los mismos.

- Por último, pensamos que esta técnica, si bien no es la panacea en los tratamientos rehabilitadores del maxilar posterior atrófico, sí constituye una herramienta válida que el cirujano de implantes debe manejar para otorgar la mejor opción terapéutica a este tipo de pacientes con reabsorciones extremas del maxilar superior.

\section{ABSTRACT}

There is a high failure rate in implants placed in the posterior atrophic maxilla. That also occurs with those placed in bone type IV (thin cortical bone around poor density trabecular bone). Nevertheless, there is a higher predictability when placing implants in the pterigoid plate. These implants can help to the stability of the prosthetic system when we are treating this kind of clinic situations.

The aim of this article is to review the procedure of implant placement in the pterigoid plate. We develop the applied anatomy concepts in this area, and a surgical and prosthetic protocol for this special approach as well.

The surgical protocol includes the cortical anchorage of the implant into the pterigoid plate. To achieve this goal the fixture must be placed within an occlusal plane angulation, in such a way as make contact with the three bones: the maxilla, the palatine bone, and the pterigoid plate of the sphenoid bone.

The prosthetic protocol also includes some variations. For example, we must take the impression directly from the implant, instead of taking it from the abutment. These changes improve the biomechanic conditions of the implants. 


\section{KEY WORDS}

Dental implants, pterigoid plate, atrophic maxilla.

\section{CORRESPONDENCIA}

Mateos, Leopoldo.

Av. Eduardo Dato, $30.1^{\circ} \mathrm{D}$.

41005. Sevilla.

\section{BIBLIOGRAFÍA}

1. ALBREKTSSON, T; ZARB, G.; WORTHINGTON, P. y ERIKSSON, A.R. The long-term efficacy of currently used dental implants: A review and proposed criteria of success. Int J Oral and Maxillofacial Implants 1986; 1: 11-25.

2. CRANIN, A.N.; RABKIN, M.F.; GARFINKEL, L.A. Statistical evolution of 952 endosteal implants in humans. J Am Dent Assoc 1997; 94: 315-329.

3. BRÄNEMARK, P.I.; HANSSON, B.; ADELL, R. y cols. osseointegrated implants in the treatment of the edentolous jaw. Scand J Plast Reconstr Surg 16 (suppl), 1997.

4. ADELL, R.; LEKHOLM, V.; ROCKLER, BRÄNEMARK, P.I. A 15-year study of osseointegrated implants in the treatment of the edentolous jaw. Int J Oral Surg 1981; 10: 387416.

5.VAN STEENBERGHE, M.D.; LEKHOLM, U. y cols. The applicability of osseointegrated oral implants in the rehabilitation of partial edentulism: A prospective multicenter study on 558 fixtures. Int J Oral Maxillofacial Implants 1990; 5: 272-281.

6. LEKHOLM, U.; ZARB, G.A. Patient selection and preparation. En: Bränemark, P.I.; Zarb, G.A.; Albrektsson, T. (eds). Tissue-integrated prostheses: osseointegration in clinical dentistry. Quintessence Publishing Co. Chicago $1985 ; 199-209$.

7. HENRY, P.J.; TOLMAN, D.E.; BOLENDER, C. Aplicación de los implantes osteointegrados en el tratamiento de pacientes edéntulos parciales: Resultados de 3 años de un estudio prospectivo multicéntrico. Quintessence Int 1993; 24: 123-129.

8. JAFFIN, R.A.; BERMAN, C.L. The excesive loss of Bränemark fixtures in type IV bone : A 5 years analysis. J Periodontol 1991; 62: 2-4.

9. RIEGER, M.R. Loading considerations for implants. Oral Maxillofacial Clin North Am 1991; 3: 795-804.
10. TATUM, H. Maxillary and sinus implant reconstruction. Dent Clin North Am 1986; 30: 207-229.

11.WOOD, R.; MOORE, D. Grafting of the maxillary sinus with intraorally harvested autogenous bone prior to implants placement. Int J Oral Maxillof Implants 1988; 3: 209-214.

12. SMILER, D.; JOHNSON, P.; LOZADA, J. Y cols. Sinus lift grafts and endosseous implants. Treatment of the atrophic posterior maxilla. Dent Clin North Am 1992; 36: 151188.

13. MOY, P.; LUNDGREN, S.; HOLMES, R. Maxillary sinus augmentation : histomorphometric analysis of graft materials for maxillary sinus floor augmentation. J Oral Maxillofac Surg 1993; 51: 857-862.

14. BRÄNEMARK, P.I.; ADELL, R.; ALBREKTSSON, T. y cols. An experimental and clinical study of osseointegrated implants penetrating the nasal cavity and maxillary sinus. J Oral Maxillofac Surg 1984; 4: 497-505.

15. GARCÍA-CALDERÓN, M.; GUTIÉRREZ, J.L.; COMPAGNI, R. Tratamiento con implantes del maxilar posterior atrófico: Alternativas al injerto óseo en el seno maxilar. Periodoncia 1996; 6: 175-183.

16. KROGH, P. Anatomic and surgical considerations in the use of Osseointegrated Implants in the posterior maxilla. Oral and Maxillofacial Surgery Clinics of North America 1991; 3: 853-869.

17. NYMAN, S.; LANG, N.; BUSER, D.; BRÄGGER, U. Bone regeneration adjacent to titanium dental implants using guided tissue regeneration: a report of two cases. Int J Oral Maxillofac Implants 1990; 5: 9-14.

18. BECKER, W.; BECKER, B. Guided tissue regeneration for implants placed into extraction sockets and for implants dehiscences: surgical techniques and case reports. Int J Periodont Rest Dent 1990; 10: 377-391.

19. NEVINS, M.; MELLONIG, J.T. Enhancement of the damaged edentulous ridge to receive dental implants: a combination of allograft and the Gore-Tex membrane. Int J Periodont Rest Dent 1992; 12: 97-110.

20. SHANAMAN, R.H. The use of guided tissue regeneration to facilitate ideal prosthetic placement of implants. Int J Periodont Rest Dent 1992; 12: 257-265.

21. BALSHI, T.J.; HERNÁNDEZ, R.E.; y cols. A comparative study of one implant versus two replacing a single molar. Int J Oral Maxillofac Implants 1996; 11:372-378.

22. LANGER, B.; LANGER, L.; HERRMANN, I.; JOURNEUS, L. The wide fixture: A solution for special bone situations 
and a rescue for the compromised implant. Part I. Int J Oral Maxillofac Implants 1993; 8: 400-408.

23. GRAVES, S.L.; JANSEN, C.; SIDDIQUI, A.; BEATY, K. Implantes de diámetro superior. Indicaciones, consideraciones y resultados en un período de 2 años. Act Implantol 1995; 7: 73-80.

24. BAHAT, O.; HANDELSMAN, M. Use of wide implants and double implants in the posterior jaw: $A$ clinical report. Int J Oral Maxillofac Implants 1996; 11: 379-386.

25. TUSLANE, J.F. Implant treatment of the missing posterior dentition. En: Albrektsson, T.; Zarb, G. (eds). The Bränemark osseointegrated implant. Quintessence Books. Chicago, 1989; 103-115.

26. BALSHI, T.J. Single tuberosity osseointegrated implant support for a tissue-integrated prosthesis. Int J Periodont Rest Dent 1992; 12: 345-357.

27. BAHAT, O. Osseointegrated implants in the maxillary tuberosity: report on 45 consecutive patients. Int J Oral Maxillofac Implants 1992; 7: 459-467.

28. GRAVES, S.L. The pterigoid plate implant: a solution for restoring the posterior maxilla. Int J Periodont Rest Dent $1994 ; 14: 513-523$.

29. BALSHI, T.J.; LEE, H.Y.; HERNÁMDEZ, R.E. The use of pterigomaxillary implants in the partially edentolous patient: a preliminary report. Int J Oral Maxillofac Implants 1995; 10: 89-98.
30. PI-URGELL, J. Implantes en la región pterigomaxilar: estudio retrospectivo con seguimiento de 1 a 10 años. RCOE 1998; 3: 339-348.

31. TESTUT, L.; LATARJET, A. Tratado de anatomía humana. Salvat Editores, S.A. $9^{\text {a }}$ edición. Barcelona, 1990.

32. ORTS LLORCA, F. Anatomía humana. Editorial científicomédica. $6^{\mathrm{a}}$ edición. Barcelona, 1987.

33. LLOYD DUBRUL, E. Anatomía oral. Ediciones Doyma. $8^{\mathrm{a}}$ edición. Barcelona, 1990.

34. WILLIAMS, P.L.; WARWICK,R. Gray Anatomía. Salvat Editores, S.A. Barcelona, 1986.

35. ROHEN, J.W.; YOKOCHI, C. Atlas fotográfico de anatomía humana. Ediciones Doyma. $2^{\text {a }}$ edición. Barcelona, 1989.

36. MOORE, K.L. Anatomía. Orientación clínica. Editorial Médica Panamericana, S.A. Buenos Aires, 1982.

37. ROUVIÉRE, H.; DELMAS, A. Anatomía humana descriptiva, topográfica $\mathrm{y}$ funcional. Ediciones Masson. Barcelona, 1994.

38. FERNER, H.; STAUBESAND, J. Sobotta. Atlas de Anatomía humana. Editorial Médica Panamericana, S.A. $18^{\mathrm{a}}$ edición. Madrid, 1983. 
